\title{
Upper Arm Disability after Axillary Surgery for Early Breast Cancer
}

\author{
Karen Mbaabu ${ }^{1}$, Ronald Wasike ${ }^{2}$
}

1. Department of Surgery, The Mater Hospital, Nairobi, Kenya

2. Department of Surgery, The Aga Khan University Hospital, Nairobi, Kenya

\section{Correspondence to: Dr Karen Mbaabu; email: karenmbaabu@gmail.com}

\begin{abstract}
Background: The treatment of early breast cancer includes surgical removal of the tumor and evaluation of axillary lymph nodes. Axillary lymph node surgery is associated with upper arm morbidity. The impact of this upper arm limitation has a direct effect on the patient's quality of life. Objectives: To quantify the symptoms of upper extremity disability, and identify correlations between severity of symptoms and type of axillary surgery. Methods: An observational study of 2 groups of patients who had undergone surgery for breast cancer. Data were collected using the DASH ${ }^{\circledR}$ questionnaire and analyzed using SPSS v13®. Results: All 102 participants reported upper extremity symptoms. The mean DASH score for all participants was 51.7. Participants in the retrospective arm had a higher DASH score of 53; those in the prospective arm had a score of 47.3. Conclusion: There was no correlation between severity of symptoms
\end{abstract}

\section{Introduction}

In general, patients with early stage breast cancer undergo primary surgery to the breast (breast conserving surgery or mastectomy) and regional lymph node dissection, with adjuvant treatments composed of chemotherapy or radiotherapy.

The state of the axillary lymph nodes has a large bearing on the overall prognosis of the patient. Clinically impalpable nodes in breast cancer undergo sentinel lymph node biopsy (SLNB) to identify nodal metastasis, which, if present, will result in axillary lymph node dissection (ALND) (1,2).

Axillary surgery often results in long-standing upper arm physical impairments and functional limitations (3). Common impairments include pain, lymphedema and upper arm weakness (4). Of these, upper arm disability has been shown to affect quality of life (QOL) the most (5). Upper arm disability is defined as limitation of a previously normal functional upper extremity, extending from the shoulder to the wrist. It is a specific disease and type of axillary surgery performed. Targeted rehabilitation services should be implemented after the primary surgery.

Keywords: Early breast cancer, Axillary dissection, DASH score, Upper arm disability

Ann Afr Surg. 2020; 17(3):99-102

DOI: http://dx.doi.org/10.4314/aas.v17i3.2

Conflicts of Interest: None

Funding: None

(C) 2020 Author. This work is licensed under the Creative Commons Attribution 4.0 International License

Submitted: 30 August 2018

Revised: 30 May 2019

Accepted: 1 August 2019

Online first: 29 May 2020 entity whose impact has increased in the last 10 years $(6,7)$. Despite breast cancer being the most common cancer that affects females in Kenya, no current national figures describe the incidence and degree of postoperative upper arm disability.

Hack et al. surveyed 222 women who underwent ALND with or without radiotherapy and reported that $72 \%$ experienced arm/shoulder pain, weakness, or numbness. The authors found that the presently used "pain intensity and affect scales" independently accounted for a statistically significant portion of the variance in QOL (5). Due to the numerous options and measurable variables for assessing upper arm disability, previous studies $(4,5)$ have mostly evaluated the patient in either of 3 domains: lymphedema, physical restriction in arm movements, and quality of life.

The DASH (Disability of the Arm Shoulder and Hand) outcome measure is a 30-item self-report questionnaire designed to measure physical function and symptoms in people with any of several musculoskeletal disorders of 
the upper limb. The tool gives clinicians and researchers the advantage of having a single, reliable instrument that can be used to assess any or all joints in the upper extremity.

In most studies, the questionnaire is used to assess effectiveness of treatment to the upper limb. Gosselink et al. showed that the type of surgery and axillary irradiation influenced shoulder function in the short- and long term substantially (8). Smoot et al. (9) used the DASH questionnaire on 120 women who had undergone axillary surgery for breast cancer. The questionnaire was used along with other objective measures such as grip strength using a dynamometer, goniometer measurement of range of movement as well as arm volume changes to establish lymphedema.

In our study, we used this tool as a one-off assessment for each patient. For the purposes of this study, the DASH questionnaire was not used to assess the effectiveness of an intervention. Instead, we used it because of its validated, non-invasive nature of assessing arm symptoms. We analyzed responses to sections of the questionnaire (domains) which are attributed to particular symptoms such as pain and quality of life.

\section{Methodology}

The study was performed in a private urban university teaching hospital. It has a 254-bed capacity, serving a catchment area of approximately 1 million patients.

The study was conducted as a cohort study. The 2 cohorts were denoted prospective and retrospective, in relation to the timing of the study. Those who had received surgery before the study were termed retrospective. We included patients who had surgery up to 2 years prior. Patients whose data were collected after the study begun were grouped as prospective.

The aims of the study were: to determine the occurrence of upper extremity disability in patients with breast cancer undergoing axillary dissection; to quantify upper arm disability using the DASH questionnaire using the disability score of patients who had undergone axillary dissection.

Our null hypothesis was that patients receiving treatment for early breast cancer do not experience significant disability in upper extremity dysfunction after axillary surgery for breast cancer.

Data were collected via a questionnaire, which collected patient specific variables. A DASH (C) questionnaire was then administered to the patient to respond to, in the presence of the principal investigator. Data were then analyzed in SPSS v 13 and association of means (using
Student's $t$ test) were calculated. Patients in the prospective arm were seen at the surgical outpatient clinic preoperatively and informed about the study. They were then recruited after giving consent.

Patients' information was entered 6 weeks postoperative. In the retrospective part of the study, patients who had undergone axillary surgery up to 2 years prior to the study were contacted from a pre-existing hospital. The patients who responded filled the DASH questionnaire at a face-to-face interview with the PI. The principal investigator also attended monthly cancer support groups within the institution where patients were also informed about the study and recruited into the retrospective arm after consenting.

Ethical approval was obtained from the institutional Ethics Review Board before starting the study. The Institute for Work and Health group gave permission to use the DASHC questionnaire for this study as per copyright agreement.

Informed consent was sought from the patients at the time of administering the questionnaire.

The inclusion criteria were: patients with early stage (stage I-II breast cancer) who had undergone axillary surgery (sentinel lymph node biopsy as well as axillary lymph node clearance) together with breast conserving surgery or modified radical mastectomy. They were above the age of 18 years and were not pregnant.

Because the DASH questionnaire does not have a validated Kiswahili version available, the study participants were required to be English speaking.

Exclusion criteria included patients with previously coexisting musculoskeletal disorders and patients who were involved in an on-going clinical trial.

Continuous and categorical data were collected from both groups including age and type of surgery (whether breast conserving or modified radical mastectomy). The DASH questionnaire was filled at the interview, with the primary investigator present to take the patient through the form.

Patient follow-up was continued as per department protocol (in the prospective group). Appropriate referrals were made to respective departments such as physiotherapy and counseling. Often, the patient's DASH score informed these referrals.

The estimated sample size using a two-sided significance level to yield a power of $80 \%$ was 102 .

The DASH questionnaire consists of a series of domains that pertain to Activities of daily living (ADL) that would be affected by: heaviness of the limb (ascribed to lymphedema), pain, peripheral neuropathy, and quality of 
life. Scores from these particular questions were totaled separately and analysis drawn from those figures.

DASH score calculation: as per the DASH questionnaire

DASH scoring formula $=$

([(sum of $n$ responses) $/ n]-1 *(25)$

where $n$ represents the number of completed items.

\section{Data analysis}

Data was input into SPSS V.13 on completion of the study. Descriptive statistics (proportions and means) were used to describe the demographic characteristics of the participants. Other variables (type of surgery, whether sentinel lymph node biopsy or axillary lymph node dissection) were tabulated. The differences in the proportions were estimated using chi-square tests, and $p$ value of 0.05 used for significance.

Primary data analysis determined any associations between the disabilities to the type of surgery (whether SLNB or ALND). A logistic regression model was used to determine this association. Further analysis was carried out to determine the associations between components of the DASH questionnaire to the total DASH score to further characterize the type of disability the patient experienced.

\section{Results}

A total of 102 participants were enrolled into this study; $75(73.5 \%)$ were recruited in the retrospective arm and 27 $(26.5 \%)$ in the prospective arm of the study (Table 1).

The mean DASH score for all participants was 51.7 $(\mathrm{SD}=17.3)$. Participants in the retrospective arm had a higher DASH score of $53(\mathrm{SD}=17.7)$ than the prospective arm 47.3 ( $\mathrm{SD}=15.9)$. This difference was however not statistically significant $(\mathrm{p}=0.128$; Student $\mathrm{t}$-test). There was no association between the MRM procedure and the selected DASH domain disabilities of quality of life, neuropathy and lymphedema (Table 2). Similarly, there was no association between SLNB procedure and the selected DASH domain scores (Table 3).

Table 1. Distribution of procedures on participants

\begin{tabular}{|c|c|c|}
\hline \multirow[b]{2}{*}{ Procedure } & \multicolumn{2}{|c|}{ Study arm } \\
\hline & Retrospective & Prospective \\
\hline Breast conserving surgery & $9(56.2 \%)$ & $7(43.8 \%)$ \\
\hline $\begin{array}{l}\text { Modified radical } \\
\text { mastectomy }\end{array}$ & $66(76.7 \%)$ & $20(23.3 \%)$ \\
\hline $\begin{array}{l}\text { Sentinel lymph } \\
\text { biopsy }\end{array}$ & $11(52.4 \%)$ & $10(47.6 \%)$ \\
\hline
\end{tabular}

Table 2. Association between breast surgery and selected domains in the DASH scores

\begin{tabular}{cccccc}
\hline & $n$ & Mean & SD & MD & $p$ value \\
\hline $\begin{array}{c}\text { Dash score } \\
\text { MRM }\end{array}$ & 86 & 51.57 & 17.660 & 0.992 & 0.834 \\
BCS & 16 & 52.67 & 15.940 & - & \\
$\begin{array}{c}\text { Quality of life } \\
\text { MRM }\end{array}$ & 86 & 8.36 & 5.418 & 0.510 & 0.703 \\
BCS & 16 & 8.87 & 4.973 & & \\
Neuropathy & & & & & \\
MRM & 86 & 4.30 & 2.042 & - & 0.646 \\
BCS & 16 & 4.56 & 2.250 & 0.216 & \\
Lymphedema & & & & & \\
MRM & 86 & 26.12 & 11.670 & 0.877 & 0.775 \\
BCS & 16 & 25.25 & 8.750 & & \\
\hline SD - Standard deviation & \multicolumn{7}{c}{ MD - Mean difference } \\
MRM - Modified radical mastectomy BCS - Breast conserving surgery
\end{tabular}

Table 3. Association between axillary surgery and selected domains in the DASH scores

\begin{tabular}{llllll}
\hline $\begin{array}{l}\text { Selected } \\
\text { domain }\end{array}$ & $n$ & Mean & SD & MD & $P$ value \\
\hline $\begin{array}{l}\text { Dash score } \\
\quad \text { SLNB }\end{array}$ & 21 & 49.14 & 14.48 & - & 0.45 \\
$\quad \begin{array}{l}\text { ALND } \\
\text { Lymphedema }\end{array}$ & 81 & 52.39 & 18.08 & 3.255 & \\
$\quad$ SLNB & 21 & 23 & 8.23 & -3.76 & 0.45 \\
$\quad$ ALND & 81 & 26.76 & 11.89 & & \\
Neuropathy & & & & & \\
$\quad$ SLNB & 21 & 4 & 1.944 & -.432 & 0.40 \\
$\quad$ ALND & 81 & 4.43 & 2.312 & & \\
Pain & & & & & \\
$\quad$ SLNB & 21 & 4.38 & 1.80 & -594 & 0.32 \\
$\quad$ ALND & 81 & 4.97 & 2.54 & & \\
Quality of life & & & & & \\
$\quad$ SLNB & 21 & 7.28 & 2.84 & - & 0.23 \\
ALND & 81 & 8.74 & 5.28 & 1.455 & \\
\hline
\end{tabular}

SLNB - Sentinel lymph node biopsy; ALNB - Axillary lymph node biopsy

\section{Discussion}

The use of the DASH questionnaire offers a useful objective tool for assessing upper arm disability. Aasheim et al. (11) established that the normal population would have an average score of up to 22 (the higher scores from older patients). Thus, any score above that is considered indicative of upper arm limitation.

From our study, the mean DASH score was high; most patients' scores were within 50-60. The highest DASH score of 100 was from a long-term survivor who had more severe grade lymphedema with significant upper extremity impairments.

Results from our study indicate that patients who had undergone axillary dissection surgery had a high DASH score. This further emphasized the work done by Johansen et al. (12) who demonstrated a higher disability in a subgroup of axillary dissection patients.

While the DASH questionnaire is most commonly used for describing improvement in upper interventions as a before and after tool, our study used it as a one-off 
assessment tool. We also used it to assess the scores given for individual impairments, particularly quality of life, neuropathy and lymphedema. The choice of evaluating these domains was based on similar studies that describe these as the most common concerns for breast cancer survivors. It must be noted however, that while the use of DASH (total) scores is validated, our study of the individual component scores is not validated. Nevertheless, the scores will help in the design of future studies.

We analyzed responses on the DASH questionnaire that enquired about: upper arm heaviness (questions 6-16), neuropathy (questions 26, 27), pain (questions 24, 25) and quality of life (questions 22,23,29,30) as the main symptoms following axillary surgery.

While there were no significant correlations between the domains of neuropathic symptoms, pain and quality, the temporal effect may cause an increase in the number of symptoms picked by this data tool.

Because the interviews were conducted based on the availability of the patients, most of the non-responders had cited unavailability to participate in the study. This markedly reduced the timely accrual of data.

Patients on the retrospective arm were significantly more than those enrolled in the prospective arm. This was probably due to the higher number of cancer survivors that were reachable either by phone or at the clinic. Due to the face-to-face nature of the administration of the questionnaire, the Hawthorne effect may have come into play (13). However, it was necessary to administer the questionnaire in this manner, as some of the respondents were not familiar with the DASH questionnaire format.

\section{Conclusion}

Most post-breast cancer patients undergo challenges associated with neuropathic symptoms. Further studies are necessary to define interventions on the same.

\section{References}

1. Sclafani LM, Baron RH. Sentinel lymph node biopsy and axillary dissection: aAdded morbidity of the arm, shoulder and chest wall after mastectomy and reconstruction. J Cancer. 2008;14(4):216-22.

2. Beaulac SM, McNair LA, Scott TE, et al. Lymphedema and quality of life in early stage breast cancer. Arch Surg. 2002;137(11)1253-1257.

3. Erwetz M, Jensen AB. Late effects of breast cancer treatment and potentials for rehabilitation. Acta Oncol. 2011;50:187193.

4. McNeely ML, Binkley JM, Pusic AL, et al. A prospective model of care for breast cancer rehabilitation: Postoperative and post-reconstructive issues. Cancer. 2012;118(8 Supplement): 2226-2236.

5. Hack TF, Cohen L, Katz J, et al. Physical and psychological morbidity after axillary lymph node dissection for breast cancer. J Clin Oncol. 1999;17(1):143-149.

6. Hayes SC, Johansson K, Stout NL, et al. Upper-body morbidity after breast cancer: Incidence and evidence for evaluation, prevention, and management within a prospective surveillance model of care. Cancer. 2020;118(S8):2237-2249.

7. Engel J, Kerr J, Schlesinger-Raab A, et al. Predictors of quality of life of breast cancer patients. Acta Oncol. 2003;42(7):710 718.

8. Gosselink R, Rouffaer L, Vanhelden P, et al. Recovery of upper limb function after axillary dissection. J Surg Oncol. 2003;83(4)204-211

9. Smoot B, Wong J, Cooper B, et al. Upper extremity impairments in women with or without lymphedema following breast cancer treatment. J Cancer Surviv. 2010;4(2)167-178. http://www.dash.iwh.on.ca/about-dash (last retrieved Feb 2019).

11. Aasheim T, Finsen V. The DASH and the QuickDASH instruments. Normative values in the general population in Norway. J Hand Surg Eur. 2014;39(2):140-144.

12. Johansson K, Holmström $H$, Nilsson I, et al. Breast cancer patients' experiences of lymphedema. Scand. J. Caring Sci. 2003; 17(1):35-42.

13. Hawthorne effect: What is Hawthorne effect? MBA Learner 2018-02-22. 